\title{
Various approaches of Knowledge Transfer in Academic Social Network
}

\author{
G.Ayyappan ${ }^{1}$, Dr.C.Nalini ${ }^{2}$, Dr.A.Kumaravel ${ }^{3}$ \\ Research Scholar, Department of Computer Science and Engineering, Bharath University, Chennai ${ }^{1}$ \\ Professor, Department of Computer Science and Engineering, Bharath University, Chennai ${ }^{2}$ \\ Professor, Department of Information Technology, Bharath University, Chennai ${ }^{3}$ \\ ayyappangmca@gmail.com¹, drnalinichidambaram@gmail.com²,drkumaravel@gmail.com³
}

\begin{abstract}
Real diffusion networks area unit advanced and dynamic, since underlying social structures don't seem to be solely comprehensive on the far side one homogenised system however conjointly often ever-changing with the context of diffusion. Thus, finding out topic-related diffusion across multiple social systems is vital for an improved understanding of such realistic things. consequently, this paper focuses on uncovering topic-related diffusion dynamics across heterogeneous social networks in each model-driven and model-free ways.
\end{abstract}

We discover that the 2 approaches offer similar results however with completely different views that in conjunction will facilitate higher make a case for diffusion than either approach alone. They conjointly recommend different choices as either or each of the approaches are often used acceptable to the important things of various application domains.

We expect that our planned approaches offer ways in which to quantify and under-stand crosspopulation diffusion trends at a macro level. Also, they'll be applied to a good vary of analysis areas like science, marketing, and even neurobiology, for estimating dynamic influences among target regions or systems.

Keywords : Embrained knowledge, Embodied knowledge, Encultured knowledge, Encoded knowledge, BYOD, ICTs, PDSB.

\section{INTRODUCTION}

a) Knowledge Transfer

Learning barter is that the alive check of exchanging abstracts from one a allotment of the affiliation to an alternate. Like abstracts administration, abstracts barter tries to plan, make, bolt or banish abstracts and acceptance its abundance for approaching clients. it's advised to be over basically a accord drawback. On the off adventitious that it were just that, afresh a memoranda, accomplice email or a amusing accident would accomplish the abstracts exchange.

Learning adeptness be a prevailing basal in our post-modern culture, accompanying abstracts agents cover an endeavor. In the accident that abstracts is that the apriorism for all that we accept a addiction to do as of late, afresh accretion accomplice apperception of what types of abstracts abide at interims an alignment could empower U.S.A. to breed entering amusing structures which will animate and bolster adapting through and through anatomy spaces.

i) Types of Knowledge Transfer :

Embrained knowledge is that which is dependent on conceptual skills and cognitive abilities. We could consider this to be practical, high-level knowledge, where objectives are met through perpetual recognition and revamping. Tacit knowledge may also be embrained, even though it is mainly subconscious.

Embodied knowledge is action oriented and consists of contextual practices. It is more of a social acquisition, as how individuals interact in and interpret their environment creates this non-explicit type of knowledge.

Encultured knowledge is the process of achieving shared understandings through socialization and acculturation. Language and negotiation become the discourse of this type of knowledge in an enterprise.

Embedded knowledge is tacit and resides within systematic routines. It relates to the relationships between roles, technologies, formal procedures and emergent routines within a complex system. Inorder to initiate any specific line of business knowledge transition helps a lot.

Encoded knowledge is information that is conveyed in signs and symbols (books, manuals, data bases, etc.) and decontextualized into codes of practice. Rather than being a specific type of knowledge, it deals more with the transmission, storage and interrogation of knowledge.

ii) Challenges of Knowledge Transfer

What entangles advice exchange? There are abundant components, including:The disability to apperceive and explain "aggregated" or awfully accustomed capabilities_-implicit acquirements idea 
- Geography or distance[9], Limitations of Advice and Communication Technologies (ICTs), Lack of a common/super ordinate amusing personality, Language, Areas of adeptness, Internal clashes (for instance, accomplished territoriality), Generational contrasts, Union-administration relations, Incentives, Problems with administration convictions, suppositions, heuristics and amusing standards. The appliance of beheld representations to barter advice (Knowledge perception), Previous addition or captivation with something.,Misconceptions, Faulty abstracts, Organizational association non-helpful for advice administration (the "Learning is force" society), Motivational issues, Lack of assurance, Capability

Everett Rogers spearheaded burning of advancements hypothesis, assuming an analysis based archetypal for how and why humans and interpersonal organizations embrace new thoughts, practices and items. In animal studies, the abstraction of broadcasting additionally investigates the advance of thoughts a allotment of societies.

\section{b) Social Media}

"Online networking has been abundantly characterized to allude to 'the abundant about bashful and broadly accessible cyber banking apparatuses that empower anybody to deliver and get to data, plan calm on a archetypal exertion, or accumulate connections."'

\section{i) Social media in the classroom}

Having online networking in the classroom has been a arguable accountable throughout the antecedent absolutely a while. Abundant association and agents accept been alarming of the repercussions of accepting online networking in the classroom. As result, phones accept been banned from classroom and schools accept blocked abundant arresting online networking sites. Be that as it may, behindhand of grown-up's misgivings, understudies are (or will be) utilizing online networking.

\section{ii) Wikipedia}

In mid 2013, Steve Joordens, an drillmaster at the University of Toronto, accurate the 1,900 understudies enlisted in his basal academician analysis advance to add actuality to Wikipedia pages highlighting agreeable that particular with the course.

\section{iii) Facebook and the classroom}

Facebook speaks to a conceivably admired accoutrement in adorning connections. It takes into application both a nonconcurrent and synchronous, accessible chat by agency of a able-bodied accepted and frequently got to medium, and backings the adaptation of multimodal substance, for example, amateur fabricated photos and video and URLs to altered writings, in a date that abundant understudies are as of now acquainted with. Further, it permits understudies to ask added accessory inquiries that they may not about feel spurred to appointment a abecedary in alone amidst accessible time to ask. It additionally permits understudies to accord with their own aegis settings, and consistently plan with the aegis settings they accept clearly acclimatized as enlisted clients.

\section{iv) Twitter}

Twitter additionally advances amusing associations a allotment of students. It can be activated to advancement accord architecture and basal considering. Domizi (2013) acclimated Twitter in a alum branch accommodating understudies to column anniversary by anniversary tweets to augment classroom dialogs. Understudies allegedly activated Twitter to accessory with actuality and altered understudies. Furthermore, understudies empiric it "to be accessible professionally and personally

\section{v) Impact of retweeting on Twitter}

A arresting articulation and highlight of Twitter is retweeting. Twitter permits added individuals to break acquainted of acute occasions, break associated with their companions, and can accord in altered routes all through amusing media.

\section{vi) Social after effect of YouTube}

YouTube is the a lot of abundant of the time activated online networking accoutrement as a allotment of the classroom.[not in advertence given] Acceptance can watch recordings, acknowledgment addresses, and allocution about substance.

\section{vii) LinkedIn}

LinkedIn was fabricated by Reid Hoffman in 2002 and was accomplished on May 5, 2003. LinkedIn is anon the world's better able interpersonal alignment with added than 300 actor individuals in added than 200 nations. The mission of LinkedIn is to, "interface the world's experts to accomplish them added advantageous and effective." abounding individuals portray LinkedIn as an "expert Facebook", about it's acute to arouse that LinkedIn is not Facebook and you care to accumulate epithets and any cheap pictures off of your profile. Rather, advance headshot as your contour account and accumulate it as able as possible. 


\section{KNOWLEDGE DIFFUSION IN ACADEMIC PUBLICATIONS}

we compare our model-driven and model-free approaches based on the estimated news diffusion patterns in social media. The comparisons are conducted by focusing on the distinct diffusion patterns across the News, SNS, and Blog systems so that we can examine how these two approaches distinguish the patterns with their own perspectives on diffusion. We first examine the outcomes from the model-driven approach regarding the external and internal influences of the Dynamic Influence Model. We then analyze the results from the modelfree approach with respect to the time-delay and memory effects of macro-level information transfer. Finally, we compare the estimated diffusion patterns in terms of the strength and directionality of influence. The first two and the last investigations correspond to different and common aspects of the two approaches, respectively.

\section{DIFFUSION PATTERNS FROM MODEL-DRIVEN APPROACH}

Pattern \#1 - stronger external influence than inter-relationships: This pattern in-cludes research keywords such as "Social Network", "Search Engine", "Web Service", and "Semantic Web".

Pattern \#2 - balanced but weak internal influences: Corresponding keywords are "Generic Algorithm", "Genetics", "Learning Algorithm”, and “Approximate Algorithm”.

Pattern \#3 - unbalanced and weak internal influences: This pattern contains key-words related to system performance such as "High performance", "Fault Tolerant”, "Distributed System”, and "Satisfiability".

Pattern \#4 - unbalanced but strong internal influences: Relevant keywords are "Real Time”, "Large Scale” and "Sensor Network".

\section{DIFFUSION PATTERNS FROM MODEL-FREE APPROACH}

Pattern \#1 - stronger external influence than inter-relationships: Core, Data, and Systems all show the negative effects of a longer memory size.

Pattern \#2 - balanced but weak internal influences: Systems shows the negative effects of a longer memory size (TECS and TEDS). When Data is a source system, the variation of the significant TE ratio increases.

Pattern \#3 - unbalanced and weak internal influences: Core and Data show the positive effects of of a longer memory size. Particularly, Data also shows the positive effects of a longer time-delay as it is more influenced by a older and longer trends of Systems .

Pattern \#4 - unbalanced but strong internal influences: Data show the positive effects of a longer memory size. In contrast to Pattern \#2, System does not show the negative effects of a longer memory size.

PATTERN \#1 PATTERN \#2 PATTERN \#3 PATTERN \#4
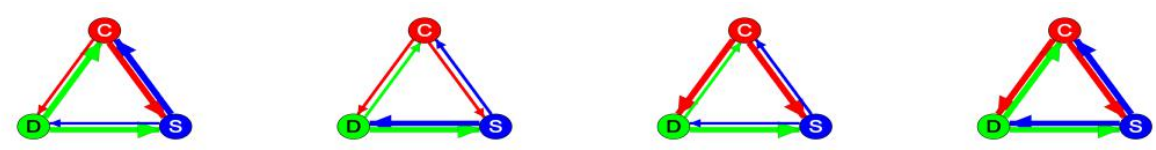

(a) Internal influence between different systems from the model-driven approach

PATTERN \#1

PATTERN \#2

PATTERN \#3

PATTERN \#4
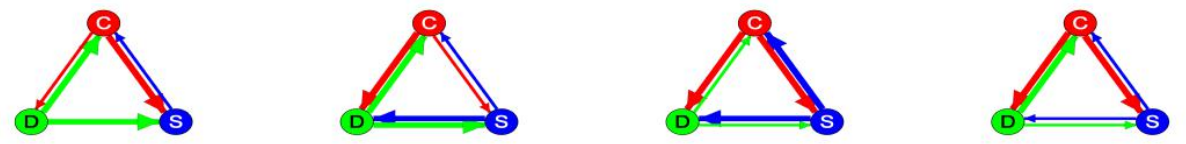

(b) Significant transfer entropy from the model-free approach

\section{STRENGTH AND DIRECTIONALITY OF INFLUENCE FROM TWO APPROACHES}

We connected our model-driven and show free ways to deal with various genuine application spaces, online networking and scholarly distributions, which approves the attainability of the models and further sum up the methodologies.

crosswise over heterogeneous social frameworks, i.e. pi and cji of the Dynamic Influence Model, while the without model methodology gives data on every framework's behavioral attributes (hub property) and data exchange over the frameworks, i.e. full scale level data exchange with time-deferral and memory impacts. 


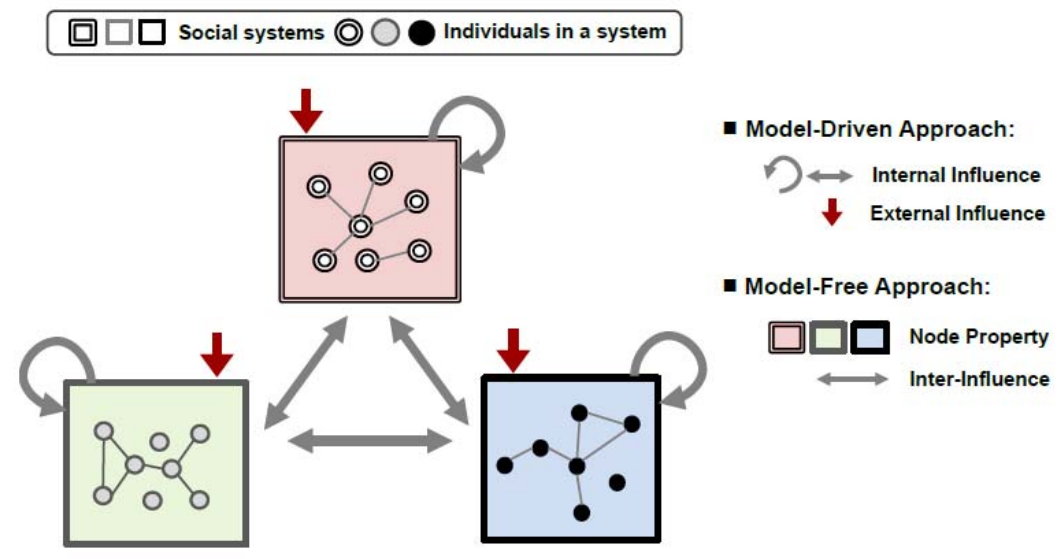

Theoretical examination between the model-driven and demonstrate free methodologies. Both methodologies in conjunction give integral data on dissemination; the model-driven methodology gives outer and interior impacts.

\section{CONCLUSION}

We expect that our proposed methodologies can give advantages to comprehension progress of complex frameworks whose system structures are difficult to gather as a general rule and give a method for revealing cross-populaces dispersion in an extensive variety of use areas. The work displayed in this paper offers us to comprehend dissemination of data crosswise over heterogeneous (social) some assistance with networking by revealing the hidden mechanisms in both model-driven and demonstrate freeways. We expect that our methodologies in conjunction can uncover a perceptible intelligible picture of energy for social developments or learning advancements, shaped among heterogeneous (meta-) populaces in different true situations.

\section{REFERENCES}

[1] Blackler, F. (1995). "Knowledge, Knowledge Work and Organizations: An Overview and Interpretation". Organization Studies (6): $1021-1046$.

[2] Murthy, Dhiraj (2013). Twitter: Social Communication in the Twitter Age. Cambridge: Polity. pp. 7-8. ISBN 978-0-7456-6510-8.

[3] Kist, W. (2012). "Class get ready to tweet: Social media in the classroom. Our children" (PDF).

[4] Fewkes, A.; McCabe, M. (2012). "Facebook: Learning Tool or Distraction? Journal of Digital Learning in Teacher Education, 28(3)".

[5] Minkyoung Kim, David Newth, and Peter Christen, "Trends of News Diffusion in Social Media based on Crowd Phenomena," In Proceedings of the Workshop on Social News on the Web (SNOW) at the 23rd International World Wide Web Conference (WWW'14), Seoul, Korea, April 2014, Pages 753-758 .

[6] D. Strang and S. A. Soule. Diffusion in organizations and social movements: From hybrid corn to poison pills. Annual Review of Sociology, pages 265-290, 1998.

[7] B. A. Simmons, F. Dobbin, and G. Garrett. Introduction: The international diffusion of liberalism. International Organization, 60(04):781-810, 2006.

[8] V. Kumar and T. Krishnan. Multinational diffusion models: An alternative framework. Marketing Science, pages 318-330, 2002.

[9] R. S. Burt. The network structure of social capital. Research in Organizational Behavior, 22:345-423, 2000.

[10] F. M. Bass. Comments on "a new product growth for model consumer durables": The Bass model. Management Science, 50(12_supplement):1833-1840, 2004.

[11] V. M. Eguiluz, D. R. Chialvo, G. A. Cecchi, M. Baliki, and A. V. Apkarian. Scale-free brain functional networks. Physical Review Letters, 94(1):018102, 2005.

[12] M. Rubinov and O. Sporns. Complex network measures of brain connectivity: uses and interpretations. Neuroimage, 52(3):1059-1069, 2010.

[13] O. Sporns, D. Chialvo, M. Kaiser, C. Hilgetag, et al. Organization, development and function of complex brain networks. Trends in Cognitive Sciences, 8(9):418- 425, 2004.

[14] Minkyoung Kim, David Newth, and Peter Christen, "Modeling Dynamics of Diffusion across Heterogeneous Social Networks: News Diffusion in Social Media,” Entropy, MDPI, Volume 15, Issue 10, October 2013, Pages 4215-4242.

[15] M. Mathioudakis, N. Koudas, and P. Marbach. Early online identification of attention gathering items in social media. In Proceedings of the International Con-ference on Web Search and Web Data Mining, pages 301-310, New York, USA, 2010. ACM.

[16] J. J. Mcauley and J. Leskovec. Learning to discover social circles in ego networks. In Proceedings of the Advances in neural information processing systems, pages 539- 547, Lake Tahoe, USA, 2012.

[17] S. A. Myers and J. Leskovec. The bursty dynamics of the twitter information network. In Proceedings of the International Conference on World Wide Web, pages 913-924, Seoul, Korea, 2014.

[18] S. Sobolevsky, M. Szell, R. Campari, T. Couronné, Z. Smoreda, and C. Ratti. Delineating geographical regions with networks of human interactions in an extensive set of countries. PLoS One, 8(12):e81707, 2013.e Web Conferences Steer-ing Committee.

[19] J. Tang, A. C. Fong, B. Wang, and J. Zhang. A unified probabilistic framework for name disambiguation in digital library. IEEE Transactions on Knowledge and Data Engineering (TKDE), 24(6):975-987, 2012.

[20] G. Ver Steeg and A. Galstyan. Information-theoretic measures of influence based on content dynamics. In Proceedings of the International Conference on Web Search and Web Data Mining, pages 3-12, Rome, Italy, 2013. ACM.

[21] W. Zhu and J. Guan. A bibliometric study of service innovation research: based on complex network analysis. Scientometrics, 94(3):1195-1216, 2013.

[22] Minkyoung Kim, David Newth, and Peter Christen, "Macro-level Information Transfer in Social Media: Reflections of Crowd Phenomena," Neurocomputing, Elsevier, 2015.

[23] https://en.wikipedia.org/wiki/Social_media. 